\title{
ZnO nano-stripes synthesized using photoelectrochemical wet etching method
}

\author{
L. S. Chuah ${ }^{1, *}$, Asmiet Ramizy ${ }^{2}$, M. A. Mahdi ${ }^{2}$, Z. Hassan $^{2}$ \\ ${ }^{1}$ Physics Section, School of Distance Education, Universiti Sains Malaysia, 11800 Minden, Penang, Malaysia \\ ${ }^{2}$ School of Physics, Universiti Sains Malaysia, 11800 Minden, Penang, Malaysia
}

Email address:

chuahleesiang@yahoo.com (L. S. Chuah)

To cite this article:

L. S. Chuah, Asmiet Ramizy, M. A. Mahdi, Z. Hassan . Zno Nano-Stripes Synthesized using Photoelectrochemical Wet Etching Method. International Journal of Materials Science and Applications. Vol. 2, No. 2, 2013, pp. 74-77. doi: 10.11648/j.jimsa.20130202.17

\begin{abstract}
To date, no approaches have been reported to fabricate the $\mathrm{ZnO}$ nano-stripes arrays on zinc foil substrate. In this method, zinc $(\mathrm{Zn})$ foil was applied as substrates. The $\mathrm{ZnO}$ nano-stripes arrays on zinc foil substrate were prepared via photoelectrochemical (PEC) wet etching method without using templates and catalysts. To prepare ZnO nano-stripes structures, the samples were dipped into a mixture of $\mathrm{HNO}_{3}$ :Ethanol $(1: 5)$ with current densities of $127 \mathrm{~mA} / \mathrm{cm}^{2}$, and subjected to external illumination from a $100 \mathrm{~W}$ lamp. The constant etch time is $30 \mathrm{~min}$. After etching, the surface morphology and the nano-stripes structure of the $\mathrm{ZnO}$ films were characterized by scanning electron microscope (SEM), energy dispersive $\mathrm{X}$-ray analysis (EDX) and X-ray diffraction (XRD). XRD pattern confirmed that the hexagonal wurtzite structure of ZnO nano-stripes were of polycrystalline structure. The optical properties of the $\mathrm{ZnO}$ nano-stripes arrays were characterized by Raman and photoluminescence spectroscopies at room temperature (RT). Micro-Raman results showed that $\mathrm{A}_{1}(\mathrm{LO})$ of hexagonal $\mathrm{ZnO}$ nano-stripes have been observed at $520 \mathrm{~cm}^{-1}$. PL spectrum peak is obvious at $368 \mathrm{~cm}^{-1}$ for $\mathrm{ZnO}$ film grown on zinc foil substrate. The PL spectrum peak position in $\mathrm{ZnO}$ nano-stripe is blue-shifted with respect to that in unstrained $\mathrm{ZnO}$ bulk $(381 \mathrm{~nm})$. This can be clarified by the approximately smaller statistical area spreading of the nano-stripes. Nanostripes $\mathrm{ZnO}$ can be used as a buffer or intermediate layer to lessen substrate-induced strain, similar to porous silicon.
\end{abstract}

Keywords: Nano-Stripes, Zno, PEC, SEM, XRD, Raman, PL

\section{Introduction}

A typical II-VI group compound semiconductor, $\mathrm{ZnO}$ is an essential technological element. A various kinds of $\mathrm{ZnO}$ nanostructures for example nanowires, nanorings, nanobows, nanotubes, nanohelices, nanorods, nanobelts, and nanoflowers have been also described over the past few years [1-4]. Recently, one-dimensional (1-D) nanomaterials particularly oriented structures have received worldwide interests due to their outstanding properties, potential applications, and high surface area [5]. Given that $\mathrm{ZnO}$ possesses a wide direct band gap $3.37 \mathrm{eV}$ at room temperature and large exciton binding energy of $60 \mathrm{meV}$, it has been the subject of abundance observation in reference to the utilize of nanostructures in the areas of electronics, electrode for dye-sensitized solar cells, gas sensors, biomedical science, green energy and photonics [6-10].

With lessen in size, novel electrical, chemical and optical properties are introduced, which are mainly conceived to be consequence of the surface and quantized energy (quantum confinement) influences. In the midst of these nanostructures, the nano-stripes have extraordinary optical, electric and magnetic characteristics, and they display promising applications in photocatalytic, chemical sensor, luminescence and field emitter [11-13]. Consequently, much struggle has been produce to the synthesis and study of their characteristics.

In this paper, we report the successful fabrication of $\mathrm{ZnO}$ nano-stripes by a simple photoelectrochemical (PEC) method. The crystalline structure, morphologies and optical properties of the $\mathrm{ZnO}$ nano-stripe were investigated.

\section{Experimental}

To prepare nano-stripes $\mathrm{ZnO}$ by photoelectrochemical (PEC) wet etching approach. For the preparation of $\mathrm{ZnO}$ nano-stripes arrays, an ordinary simple manner was as follows: A slice of zinc foil substrate $(20 \times 30 \times 0.25 \mathrm{~mm} 3)$, cleaned by absolute ethanol and followed by deionized water. 
The PEC etching of zinc ( $\mathrm{Zn}$ ) foil samples were carried out in a conventional electrochemical cell at room temperature using an unstirred of mixture of $\mathrm{HNO}_{3}$ :Ethanol (1:5) solution. Etching was also executed applying front-side illumination with the lamp and a standard electrochemical cell with a Pt counter electrode with external bias was applied between the sample and the cathode $[14,15]$. The constant etch time is $30 \mathrm{~min}$.

Samples were chemically cleaned to clear away surface contaminants and oxides before any characterization. After etching, the samples were characterized by X-ray diffraction (XRD) measurements. The morphology and composition of the samples were examined by scanning electron microscopy (SEM) combined with energy dispersive X-ray analysis (EDX).

All the optical measurements were studied at room temperature (RT). Raman spectroscopy was conducted with a micro-Raman/photoluminescence (Jobin Yvon HR800UV) system, for example, an integrated confocal microPhotoluminescence spectrometer. A He-Cd laser $(325 \mathrm{~nm})$ was used as an excitation source for PL. Micro-Raman scattering experiments were investigated by using a Jobin Yvon HR 800 UV spectrometer system. The excitation light source was the $514.5 \mathrm{~nm}$ line of Argon (Ar) ion laser beam and the incident laser power of $20 \mathrm{~mW}$ was focused [14]. Previously the $\mathrm{ZnO}$ spectrum was reported, the Raman spectroscope was calibrated and checked using a silicon substrate [16].

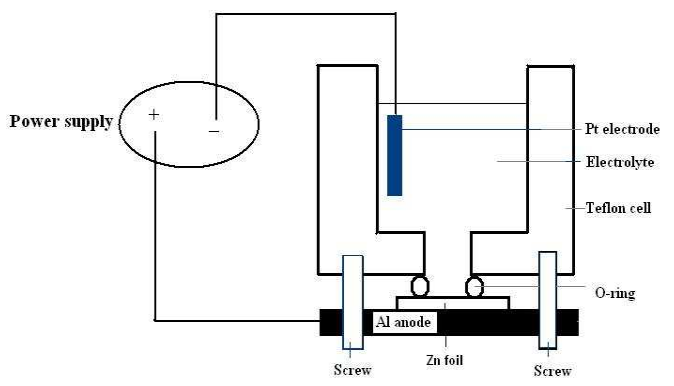

Fig. 1. The photoelectrochemical etching experimental set up used to generate $\mathrm{ZnO}$ nano-stripes arrays on zinc foil substrate.

In this work, micro-Raman scattering experiments were carried out in the $\mathrm{z}(\mathrm{x}$, unpolarized) $\bar{z}$ scattering configuration [14]. Here, the Porto's notation is used for scattering geometries with $\mathrm{z}$ parallel to wurtzite $\mathrm{c}$ axis, and (x, unpolarized) refers to the polarization of the incident and scattered light [17]. Under this configuration, the allowed zonecenter phonon modes that can be detected for wurtzite structure layer will be A1(LO), and E2(high), unless there are some disoriented microstructures [18].

\section{Results and Discussion}

The morphology of a top-view $\mathrm{ZnO}$ nano-stripe arrays grown on a zinc foil substrate is shown in Figure 2. Fig. 2(b) shows a medium-magnification SEM image of the product. It can be seen from Fig. 2, that $\mathrm{ZnO}$ nano-stripe arrays are densely but not homogenously distributed. The geometrical shape of the nano-stripe is clearly shown in Fig. 2(c) and (d). Figure 2c and d show the close-view SEM images of as synthesized $\mathrm{ZnO}$ nano-stripe structures. Figure 3 shows typical EDX results for the composition of the $\mathrm{ZnO}$ nano-stripe arrays grown on the zinc foil substrate. It discloses the presence of $\mathrm{Zn}$ and $\mathrm{O}$ as the only primary components, with a moderate oxygen defect $(\mathrm{Zn} / \mathrm{O}$ atomic ratio $=1: 1)$.

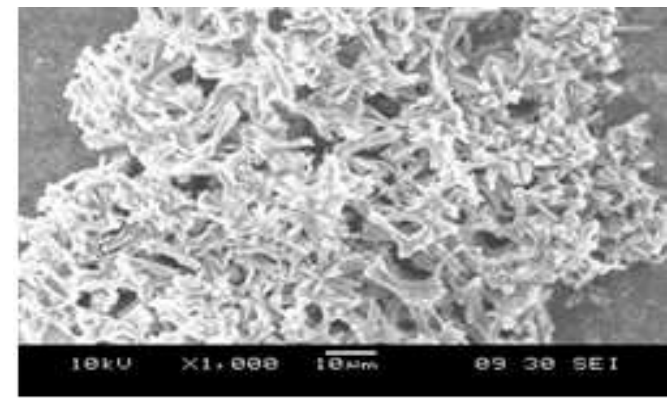

(a)

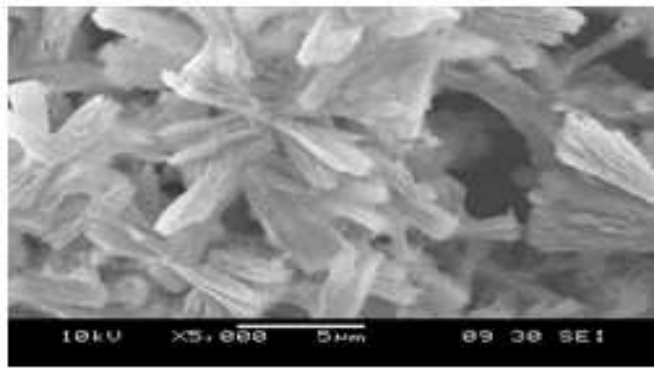

(b)

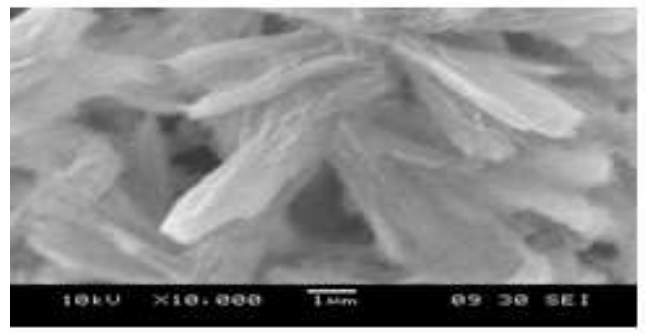

(c)

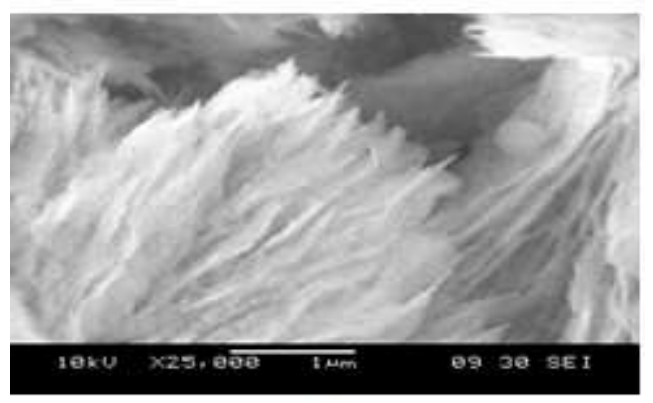

(d)

Fig. 2. SEM images of $\mathrm{ZnO}$ nano-stripe arrays on zinc foil substrate with different magnification views: (a) 1,000, (b) 5,000, (c) 10,000, and (d) 25,000 


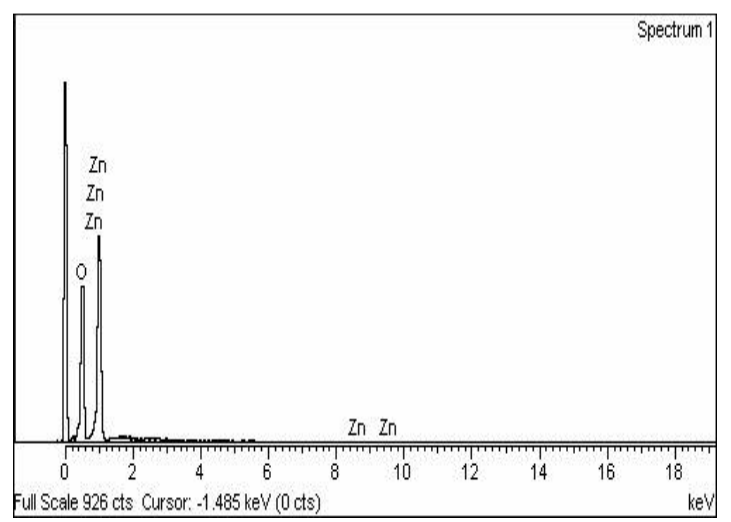

Fig. 3. The corresponding EDX pattern of the $\mathrm{ZnO}$ nano-stripe arrays on zinc foil substrate.

Figure 4 shows an XRD pattern of the as-synthesized samples. The diffraction peaks can be recorded to wurtzite $\mathrm{ZnO}$, exclude for those distinguished as $\mathrm{Zn}$ coming from the zinc $(\mathrm{Zn})$ foil substrate. Prominently, when compared with the common diffraction form, the (002) diffraction peaks are improved and much stronger than the other peaks, symbolizing the highly preferential growth of the $\mathrm{ZnO}$ nano-stripes along their c-axis, perpendicular to the $\mathrm{Zn}$ foil substrate surface.

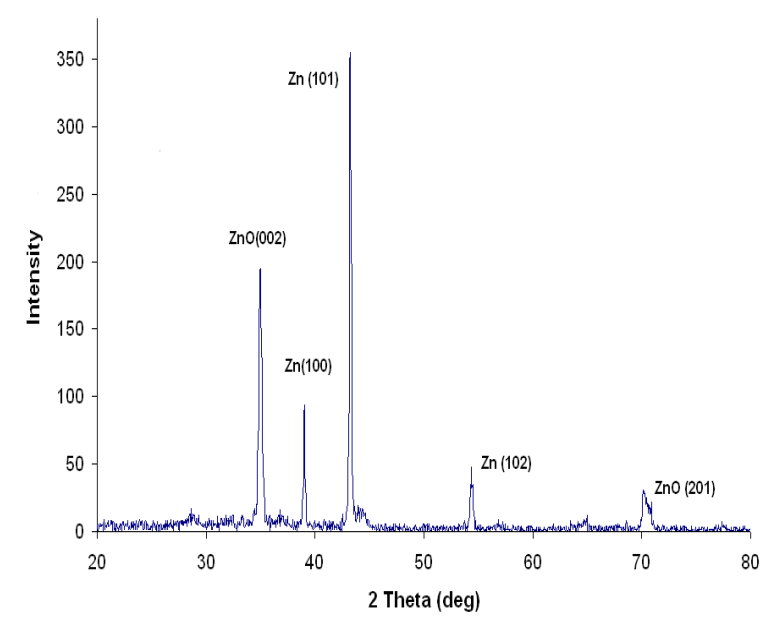

Fig. 4. X-ray diffraction pattern of the ZnO nano-stripes on Zn foil.

Figure 5 shows the Raman scattering spectrum of the $\mathrm{ZnO}$ nano-stripes. A strong peak appears at $438 \mathrm{~cm}-1$ which corresponds to the E2(H) mode of wurtzite phase of $\mathrm{ZnO}$, which is the intrinsic characteristic of the Ramanactive mode of wurtzite hexagonal $\mathrm{ZnO}$ [19], while the 530 $-620 \mathrm{~cm}-1$ peak is attributed to the presence of $\mathrm{Zn}$ interstitials [20]. The A1(LO) Raman line-shape is very sensitive to lattice disorder in $\mathrm{ZnO}$ crystals, because the nonstoichiometric defects will disrupt the long range ionic ordering [21]. The disorder will induce the phonon modes near the $\mathrm{A} 1(\mathrm{LO})$ on the phonon dispersion curve to be Raman active [22]. For $\mathrm{ZnO}$ nano-strikes, only the allowed Raman phonon modes of A1(LO) are clearly visible at 530 $\mathrm{cm}-1$ for growth on $\mathrm{Zn}$ foil.

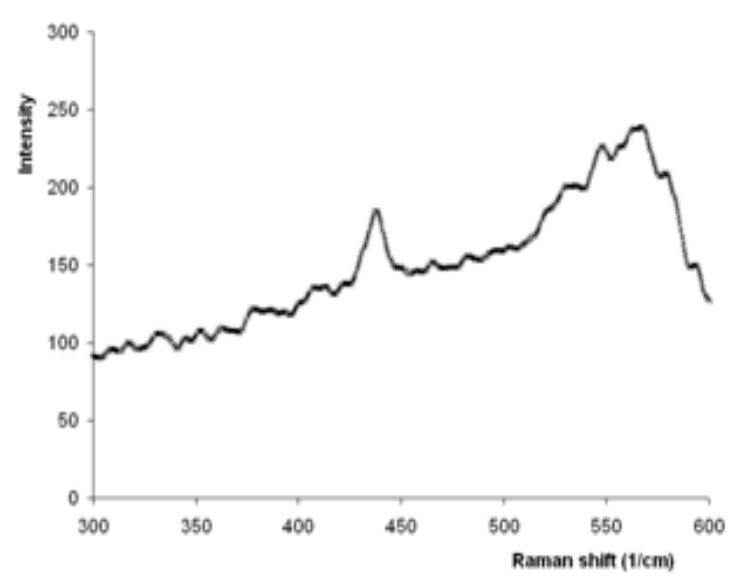

Fig. 5. Room temperature micro-Raman spectrum of $\mathrm{ZnO}$ nanostructures.

Photoluminescence (PL) spectroscopy is an efficient method for evaluating the optical properties of semiconductor materials. Figure 6 shows a typical room-temperature PL spectrum of the $\mathrm{ZnO}$ nano-stripes arrays, with a dominant emission peak centered at $368 \mathrm{~nm}$, which is corresponds to the ultraviolet (UV) emission of $\mathrm{ZnO}$ with a band gap of $3.39 \mathrm{eV}$. Many researched have reported the optical properties of $\mathrm{ZnO}$ nanoctructures, Generally, the UV emission peak of $\mathrm{ZnO}$ is attributed to the near-bandedge (NBE) emission of wide-band-gap $\mathrm{ZnO}$.

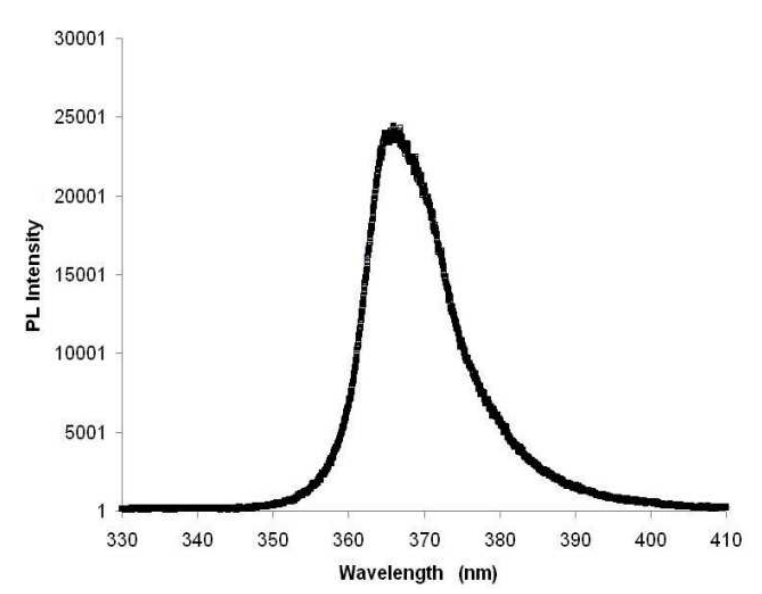

Fig. 6. The PL spectra obtained from the surfaces of the $\mathrm{ZnO}$ nano-stripes.

\section{Conclusion}

Large-scale $\mathrm{ZnO}$ nano-stripe arrays have been successfully synthesized on zinc foil by a simple and low-cost method. We synthesized the $\mathrm{ZnO}$ nano-stripes without any catalysts or templates. PL and Raman spectra show that these $\mathrm{ZnO}$ nano-stripess arrays have good optical properties. Room-temperature photoluminescence (PL) spectrum of nano-stripe arrays shows a strong emission band at about $368 \mathrm{~nm}$. Furthermore, the novel $\mathrm{ZnO}$ nano-stripes reported here may have potential applications as functional blocks in 
fabrication of optoelectronic nanodevices.

\section{Acknowledgements}

Financial support from FRGS grant and Universiti Sains Malaysia are gratefully acknowledged.

\section{References}

[1] Z. W. Pan, Z. R. Dai, Z. L. Wang, Nanobelts of Semiconducting Oxides. Science, 291 (2001) 1947-1949.

[2] Z.L. Wang, J. H. Song, Piezoelectric Nanogenerators Based on Zinc Oxide Nanowire Arrays. Science, 312, (2006) 242 246.

[3] S.H. Lee, H. J. Lee, D. Oh, S. W. Lee, H. Goto, R. Buckmaster, T. Yasukawa, T. Matsue, S. K. Hong, M. W. Cho, T. Yao, Control of the ZnO Nanowires Nucleation Site Using Microfluidic Channels. J. Phys. Chem. B 110, (2006) $3856-$ 3859 .

[4] Y. Li, F. Qian, J. Xiang, C. M. Lieber, Nanowire Electronic and Optoelectronic Devices. Mater. Today, 9, (2006) 18-25.

[5] J. M. Bao, M. A. Zimmler, F. Capasso, Broadband ZnO Single-Nanowire Light-Emitting Diode. Nano Lett. 6 (2006) $1719-1722$

[6] J. T. Hu, T. W. Odom, C.M. Lieber, Chemistry and Physics in One Dimension: Synthesis and Properties of Nanowires and Nanotube. Acc. Chem. Res. 32, (1999) 435-445.

[7] H. J. Fan, P. Werner, M. Zacharias, Semiconductor Nanowires: From Self-Organization to Patterned Growth. Small, 2 , (2006) 700-717.

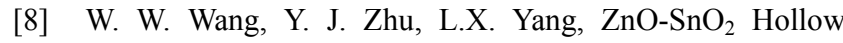
Spheres and Hierarchical Nanosheets: Hydrothermal Preparation, Formation Mechanism, and Photocatalytic Properties. Adv. Funct. Mater. 17, (2007) 59-64.

[9] C. W. Cheng, G. Y. Xu, H. Q. Zhang, Y. Luo, Fabricating $\mathrm{ZnO}$ Nanorods Sensor for Chemical Gas Detection at Room Temperature. J. Nanosci. Nanotechnol, 7, (2007) 4439-4442.

[10] ]H. Y. Yang, S.P. Lau, S. F. Yu, A. P. Abiyasa, M. Tanemura, T. Okita, H. Hatano, High-Temperature Random Lasing in ZnO Nanoneedles. Appl. Phys. Lett. 89, (2006) 011103.

[11] L. E. Greene, M. Law, D. H. Tan, M. Montano, J. Goldberger, G. Somorjai, P. D. Yang, General Route to Vertical $\mathrm{ZnO}$ Nanowire Arrays Using Textured ZnO Seeds. Nano
Lett. 5, (2005) 1231-1236.

[12] L. Vayssieres, Growth of Arrayed Nanorods and Nanowires of $\mathrm{ZnO}$ from Aqueous Solution. Adv. Mater., 15, (2003) 464-466.

[13] H. J. Fan, M. Zacharias, Manipulation of Crawling Growth for the Formation of Sub-Millimeter Long $\mathrm{ZnO}$ Nanowalls. J. Mater. Sci. Technol. 24, (2008) 589-593.

[14] L.S. Chuah, Z. Hassan, S.S. Ng, H. Abu Hassan, Porous $\mathrm{Si}(111)$ and $\mathrm{Si}(100)$ as an intermediate buffer layer for nanocrystalline InN films, Journal of Alloys and Compounds 479, (2009) L54-L58.

[15] L.S. Chuah, Z. Hassan, H. Abu Hassan, Nanoporous InN films synthesized using photoelectrochemical wet etching, IEEE International Conference on Semiconductor Electronics Proceedings (ICSE), (2006) 618-621.

[16] Y. X. Wang, X. Y. Li, G. Lu, X. Quan, G. H. Chen, Highly oriented 1-D nanorod arrays on zinc foil: direct growth from substrate, optical properties and photocatalytic activities, J. Phys. Chem. C, 112 (2008) 7332-7336.

[17] L.S. Chuah, Z. Hassan, H. Abu Hassan, Optical characterization of nanoporous $\mathrm{GaN}$ through electroless wet chemical etching, Materials Science-Poland, Vol. 26, No. 3, (2008) 609-615.

[18] L.S. Chuah, Z. Hassan, H. Abu Hassan, Optical characterization of $\mathrm{GaN}$ thin film grown on $\mathrm{Si}(111)$ by radiofrequency plasma-assisted molecular beam epitaxy, Malaysia-Japan International Symposium on Advanced Technology (MJISAT), 1-6 (2007) - on CD.

[19] J. H. Yang, J. H. Zheng, H. J. Zhai, L. L. Yang, Low temperature hydrothermal growth and optical properties of $\mathrm{ZnO}$ nanorods, Cryst. Res. Technol. 44, (2009) 87-91.

[20] P.S. Xu, Y. M. Sun, C. S. Shi, F. Q. Xu, H. B. Pan, Native point defect states in $\mathrm{ZnO}$, Chinese Physics Letters, 18 (2001) 1252-1253.

[21] [21]L.S. Chuah, M. A. Ahmad, Z. Hassan, Nanocrystalline $\mathrm{ZnO}$ film grown on porous $\mathrm{SnO} 2 / \mathrm{Si}(111)$ substrates, Composit Interfaces, 18 (2011) 627-632.

[22] L.S. Chuah, Z. Hassan, S.K. Mohd Bakhori, Optical analysis of nanocrystalline $\mathrm{ZnO}$ films coated on porous silicon by Radio Frequency (RF) Magnetron Sputtering, Composite Interfaces, 18 (2011) 441-448.

[23] L.S. Chuah, Z. Hassan, S. S. Tneh, Single crystalline ZnO nanowires by oxidizing granular zinc film, Journal of Dispersion Science and Technology, 32 (2011) 677-679. 